\title{
El discurso político indígena en América Latina
}

\author{
Águeda Gómez Suárez
}

En este artículo se pretende realizar un pormenorizado análisis en torno a los mensajes políticos construidos por algunas destacadas movilizaciones indígenas en América Latina. Para ello se ha optado por el uso del marco teórico del Frame Analysis, categoría analítica proveniente del "interaccionismo simbólico" de Goffman, interpretado hoy como un elemento central en la formación de la identidad de los actores sociales y en su "actuación social". La sistematización en varios "marcos de interpretación" de ciertas ideas significativas que conforman la matriz ideológica indígena es realizada por estos sujetos sociales con el fin de proyectar una imagen ante los demás que genere ciertas impresiones en el auditorio y que defina problemáticas, causas, soluciones y adversarios. El "éxito político" de sus propuestas y de su programa político y social va a depender de la resonancia de estos "marcos" en diferentes audiencias internas y externas.

PALABRAS CLAVE: ideología étnica, sistema cognitivo, movilizaciones indígenas, frame analysis, América Latina

This paper attempts to make a meticulous analysis of the political messages constructed by some prominent indigenous movements in Latin America. In order to do so, the author chose the theoretical framework called frame analysis - an analytical category originated by Goffman's symbolic interactionism - which today is regarded as a central element in the identity formation of social actors and their "social performance". The systematization of certain significant ideas that shape the indigenous ideological matrix into several "frameworks of interpretation" is performed by these social subjects with the purpose of projecting unto others an image that generates certain impressions in their audience and defines a set of problems, their causes, their solutions and the adversaries. The "political success" of their proposals and political program depends on the resonance that these "frameworks" can achieve with different internal and external audiences.

KEYWORDS: ethnic ideology, cognitive system, indigenous movements, frame analysis, Latin America

ÁGUEDA GÓmEZ SUÁREZ: Facultad de Ciencias de la Educación (Ourense), Universidad de Vigo, España. agueda@uvigo.es

Desacatos, núm. 22, mayo-agosto 2007, pp. 215-228.

Recepción: 29 de abril de 2005 / Aceptación: 31 de enero de 2006 


\section{INTRODUCCIÓN}

$\mathrm{E}$ n las últimas décadas se asiste a la irrupción de los movimientos indígenas como actores sociales y políticos del contexto latinoamericano que se están convirtiendo en sujetos activos del cambio histórico.

En los países de América Latina estos movimientos sociales se han deslizado de ser actores marginales de sus escenarios nacionales hacia su conversión en agentes protagonistas de la sociedad civil. La emergencia de los movimientos indígenas ha generado importantes avances normativos en el reconocimiento de los derechos colectivos de los pueblos en los terrenos nacional e internacional.

El movimiento indígena zapatista de México, el alzamiento indígena en Ecuador que precipitó la dimisión del presidente de ese país, las nuevas tendencias electorales en Perú, las manifestaciones indígenas en Brasil contra la celebración del "descubrimiento" del país, las movilizaciones populares en Bolivia que han conseguido alzar como que se está incrementando la participación ciudadana de la población indígena.

Grupos indígenas relativamente pequeños, cuando no insignificantes, suscitan de modo inesperado notables movimientos reivindicativos persistentes que logran modificar la historia de una región. Las causas que han inducido a los movimientos indígenas a optar por coordinar sus acciones colectivas no están vinculadas a la regia y tenaz identidad colectiva que comparten sus habitantes, derivado "natural" de las esencias culturales de este pueblo, sino a otra serie de factores externos e internos, objetivos y subjetivos, que ha favorecido la construcción política consciente de este movimiento (Stavenhagen, 1996: 93; Gurr, 1995: 4)

Si pese a las condiciones de aislamiento, pobreza, crisis demográfica y exclusión sociopolítica, los movimientos indígenas han conseguido alcanzar sus objetivos políticos y reforzar su presencia internacional y nacional, es necesario explicar qué factores determinaron este hecho paradójico. Este artículo intenta dar respuesta a esta interrogante por medio del estudio de la "variable discur- siva", uno de los principales factores que han intervenido en este hecho, a partir de las investigaciones realizadas por la autora (Gómez, 2001, 2004; Gómez et al., 2004) y otras investigaciones actuales en curso aún no publicadas.

\section{EL ANÁLISIS DEL DISCURSO POLÍTICO INDÍGENA: LOS “MARCOS DE INTERPRETACIÓN"}

Hasta la actualidad, el estudio de los movimientos sociales se ha caracterizado por la polarización de enfoques, lo que se deriva de las diferentes concepciones sobre los mismos (Cohen y Arato, 2000). Se está generando un consenso metodológico y analítico sobre la necesidad de evaluar los procesos colectivos de interpretación, atribución y construcción del discurso ideológico por medio del "análisis de marcos" o frame analysis ${ }^{1}$. Este sistema teórico considera que las colectividades se organizan y tienden a definir estratégica y conscientemente una situación dada, a través de "procesos enmarcadores" que favorecen la interpretación de su realidad con el fin de legitimarse y, en consecuencia, movilizar a los principales protagonistas a encabezar una acción colectiva que responda a sus intereses y objetivos más sustantivos (Tarrow, 1997). Esta categoría analítica —el frame - proveniente del "interaccionismo simbólico" de Goffman (1974), se interpreta hoy como un elemento central en la formación de la identidad de los actores sociales y en su "actuación social” (performance), con el fin de proyectar una imagen ante su auditorio y ante sí mismo.

De acuerdo con algunos de los principales teóricos de esta corriente, hay tres variables que generan y determinan los "procesos de enmarcamiento" construidos por los propios movimientos sociales. Por un lado, el elemento de "injusticia", que parte de una hot cognitions o cuestión caliente que provoca sentimientos de indignación. Otro es el elemento de la "eficacia" o valoración de las probabilidades de éxito que el movimiento realiza sobre

${ }^{1}$ Véase Goffman, 1974; McAdam, McCarthy y Zald (1999); Tarrow (1997); Snow, Hunt y Benford (1993); Ibarra y Tejerina (1998) y Gerhards (1995). 


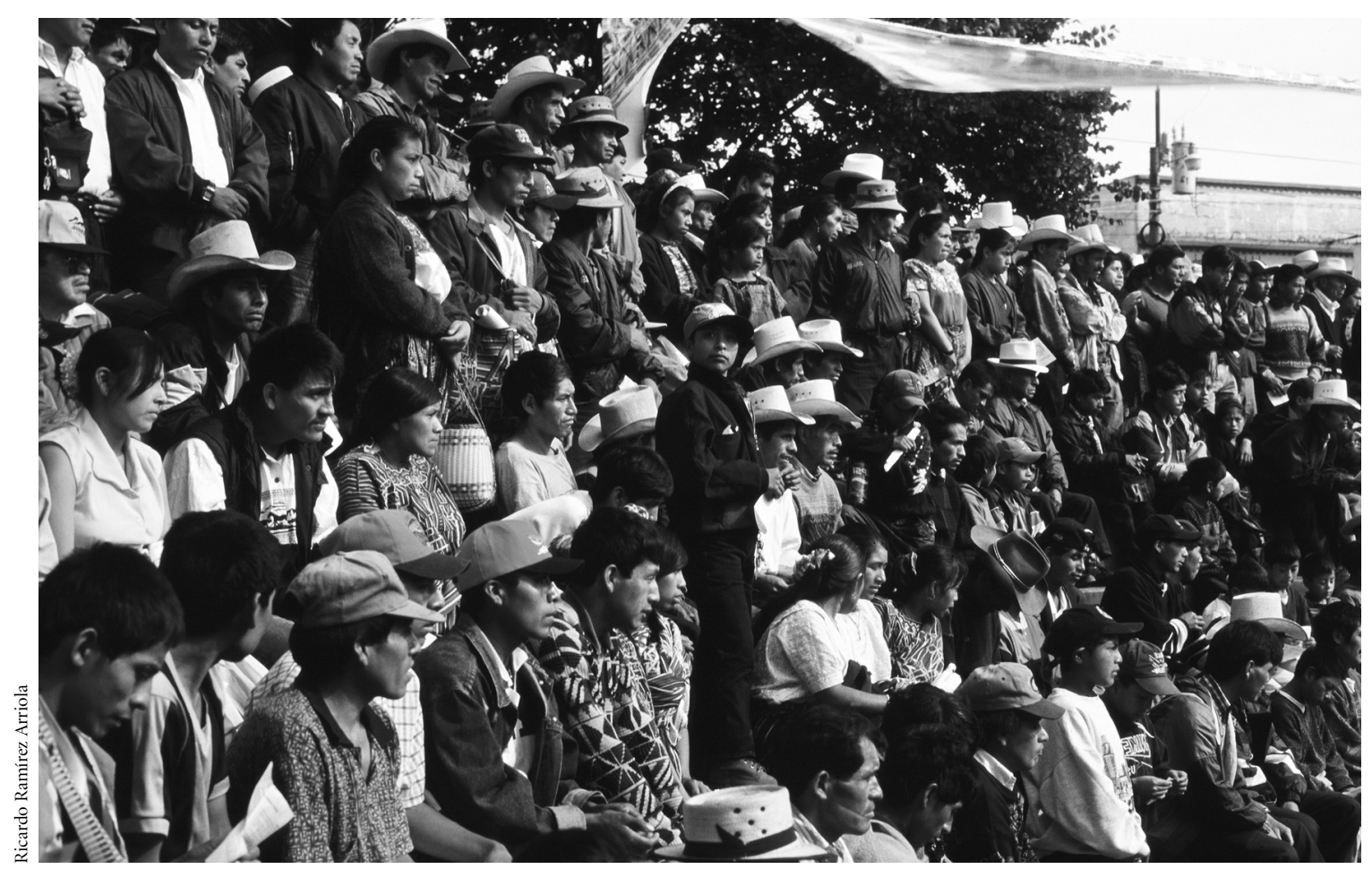

Asamblea popular, El Quiché, Guatemala, 1999.

la consecución de sus objetivos. Por último, el elemento identitario del propio actor social que ayuda a diferenciar el "nosotros" de los "ellos" (Gamson, 1990; Snow, Hunt y Benford, 1993).

Para el análisis de los marcos de interpretación indígena se van a utilizar los conceptos de dimensión y estrategia de enmarcamiento, elaborados de forma metodológica por Gerhards (1995), a partir de las aportaciones de Snow, Hunt y Benford (1993).

La argumentación que a continuación se desarrolla intenta describir las dimensiones y estrategias de enmarcamiento centrales en el discurso ideológico del movimiento indígena y en sus proclamas legitimadoras de objetivos, intereses e ideologías, a partir de los documentos y declaraciones públicas de algunos de sus dirigentes (Gómez, 2001, 2004; Gómez et al., 2004). Posteriormente se relata el momento en el que se genera el "proceso de politización" de esas estructuras culturales y se emprende el diseño de los cuatro marcos: el "identitario", el "territor- ial-ambiental", el "étnico-cultural" y el "globalicrítico". Finalmente se aborda el estudio del impacto de los mismos en sus audiencias, valorando los alineamientos y resonancias que se originan, para acabar detallando el discurso ideológico de la acción colectiva indígena y sus rendimientos, victorias y conquistas políticas.

\section{ESQUEMAS COGNITIVOSY SIMBÓLICOS. STOCK CULTURAL E IDENTIDAD FRACTURADA}

Los procesos de enmarcamiento se apoyan en las estructuras cognitivas y simbólicas, denominadas "paquetes de dispositivos" por Klaus Eder (1992), propias del "stock cultural” de cada colectividad (Zald, en McAdam et al., 1999). En este caso, la intersubjetividad común indígena descansa principalmente en el capital económico, social y cultural indígena (Bourdieu, 1988), lo que ha generado unos "sistemas de disposiciones" (Máiz, 1995), prescri- 


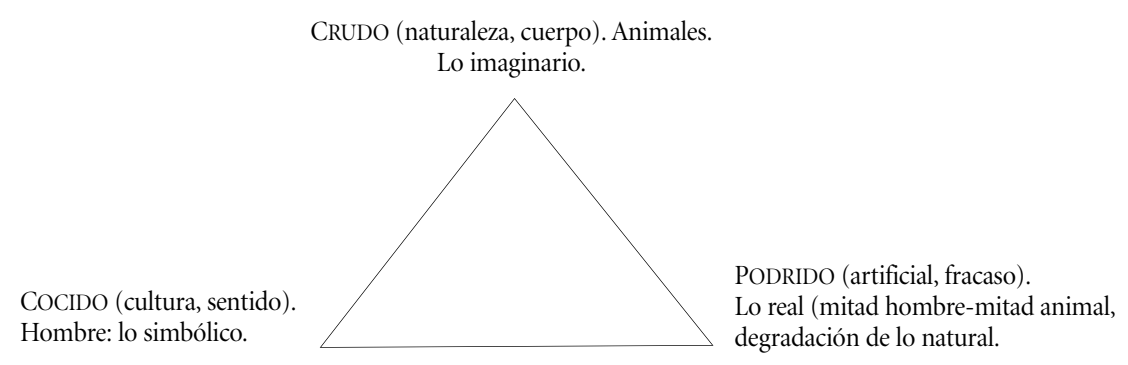

tos por sus rasgos culturales y étnicos: lengua, organización, sistema económico, estructura social, producción material, creencias, ritos, costumbres, tradiciones, etc. Este background cultural configura un marco de interpretación básico y es empleado por el movimiento como un mecanismo para enmarcar la realidad de un modo alternativo frente a la intersubjetividad hegemónica.

Los indígenas conciben al universo como una gran unidad, en la que todos los elementos están vinculados y reles garantiza el uso sostenible de los recursos naturales necesarios para la supervivencia de esta colectividad humana. Todos los entes orgánicos e inorgánicos del mundo constituyen formas de vida conscientes tuteladas por su "espíritu" o "dueño" con una actividad voluntaria animada por un alma antropomórfica que los protege y cuida.

En algunas cosmovisiones indígenas, entre el hombre y el mundo animal, entre lo cultural y lo natural, existe un continuum analógico que se manifiesta en muchas de las creencias, mitos y narraciones orales. En esta dialéctica de lo sagrado se conciben a la "criatura" y el "cosmos" subsumidos en la misma representación simbólica que desencadena una "antropomorfización de la naturaleza". En la cosmogonía indígena, los fenómenos de la naturaleza adquieren carácter místico, divino y trascendental. Si se aplica un análisis sémico levistraussiano (Lévi-Strauss, 1964; Pereña, en Delgado y Gutiérrez, 1999: 470), sobre todo a algunos de los elementos de la tradición oral indígena, la clasificación de sus "seres" míticos se puede triangular dialécticamente (gráfica 1).

En este background étnico-cultural, al que se ha deno-

minado "precondiciones étnicas", se perfila una identidad semiconsciente, precaria y de baja intensidad, producto de los procesos de fractura sociocultural y de la precariedad económica y en una representación débil y difusa de su identidad política. Esto les ha conferido peculiaridades especiales a la respuesta y a las estrategias organizativas y políticas de sus manifestaciones.

Las identidades colectivas, inicialmente segmentadas, pueden llegar a convertirse en identidades asentadas si consiguen generar alineamientos fuertes entre sus seguidores y si logran institucionalizar su acción colectiva y su "mensaje" grupal (Tilly, en McAdam et al., 1999). Es lo que va a pretender formalizar el pequeño núcleo de "intelectualidad" indígena ${ }^{2}$ en un momento histórico concreto, que a continuación se relata.

\section{EL MOMENTO DE LA "LIBERACIÓN COGNITIVA”. ¿CÓMO, CUÁNDOY POR QUÉ?}

Hay dos procesos de construcción que entran en juego cuando se analizan las identidades de los grupos étnicos, y que corresponden a cada una de las fases ontológicas de constitución de las mismas. El primer proceso de transformación articula el "bagaje cultural" de un pueblo con su "identidad étnica" imaginada (Anderson, 1993).

\footnotetext{
${ }^{2}$ En muchos casos, sólo pequeños núcleos de la burguesía local indígena han perfilado el significado de una identidad política indígena, a raíz de los legados políticos heredados de las organizaciones de cooperación al desarrollo, agencias gubernamentales, la Iglesia y otras organizaciones de ayuda al desarrollo.
} 
El segundo proceso se fundamenta en una operación de racionalización política, de naturaleza lógica y categórica (García Selgas, en Delgado y Gutiérrez, 1999), en la que se articula esta identidad étnica con la identidad política consciente, autorreflexiva y racional, que hace referencia a las orientaciones de su proyecto político. El salto de la identidad étnica a la identidad política se genera por medio de un proceso de liberación cognitiva (McAdam et al., 1999)

La construcción de la emergente identidad política indígena aparece a raíz de los procesos históricos de cambio que repercuten en el clima cultural preexistente entre la población, y que van a actuar como "ventana política" (Gamson y Meyer, 1999: 397) para la producción de nuevos marcos. En el caso de estos pueblos, el cambio del clima cultural se suscita durante la década de 1980, a partir de la llegada de la ola democratizadora en toda América Latina, acompañada del desembarco de organismos intergubernamentales, supranacionales y de organizaciones no gubernamentales trasnacionales de ayuda al desarrollo.

La introducción por parte de estas agencias de cooperación al desarrollo de una matriz cultural política "modernizadora” en las comunidades indígenas es lo que impulsa la transformación de sus discursos tradicionales hacia significados e interpretaciones ideológicas que se cubren de nuevas formas de legitimación, objetivos, intereses y prácticas.

La construcción de la identidad política indígena ha estado pilotada por la élite intelectual indígena autóctona, con ayuda de los "aliados" con los que ha contado a lo largo del tiempo. Es a través de la generación de un proceso de enmarcamiento que se sistematiza la concepción de esta identidad como práctica político-ideológica.

\section{MARCO PRINCIPALY MARCOS DERIVADOS. DE LA IDENTIDAD A LA IDEOLOGÍA. MEMORIA, PRAXISY UTOPÍA}

Para el análisis de los marcos de interpretación aplicados al discurso indígena se van a emplear los conceptos operacionalizados por Gerhards (1995), a partir de las apor- taciones de Snow y Benford (1993) y expuestos en el epígrafe teórico de este texto.

Con el objeto de sistematizar la información extraída de los mensajes indígenas, se distinguen tres frames principales en la estructura discursiva que nos ocupa. En un primer momento, se considera un marco de interpretación modelado en torno a la identidad, que denominaremos frame identitario; en un segundo momento se distingue el frame territorial-ambiental; el "marco": el frame étnico-cultural, y finalmente el frame globalicrítico.

El primer frame indígena denominado marco identitario (gráfica 2, p. 220) incide en un eje central: la constitución de la matriz básica en el que se apoya y legitiman las reivindicaciones de su movimiento. Este marco coloca en su médula la politización de la identidad social (self identity). La construcción de una identidad política ha resultado ser una de las estrategias más socorridas por los diferentes colectivos étnicos como táctica para definir sus reivindicaciones y sus definiciones del mundo (Stavenhagen, 1996; Gurr, 1995).

En la lengua autóctona, la autodenominación de un grupo como mayas, tawahkas, aymaras o yaquis, se traduce habitualmente como "primeros hombres o primogénitos", por lo que se deduce que la concepción identitaria tradicional ostenta una esencia etnocéntrica que los sitúa como pueblo en el centro del universo y de la humanidad. La identidad indígena aparece encarnada como una particularidad ya dada, como una entidad cuya posesión define al sujeto con una categoría plena y absoluta (Guerra, en León y Zemelman, 1998). Para la narrativa indígena suele siempre existir un tiempo pasado, una especie de pasado esplendoroso y de "edad de oro", en el que estos pueblos se extendían por todo el vasto territorio que los envuelve. Posteriormente, según sus narraciones típicas, entran en un fatal declive originado por la acción de ciertos agentes externos (colonizadores, empresas, etc.) y por las catástrofes naturales (huracanes, inundaciones, epidemias, etc.) que los abocó a una crítica situación de "riesgo de extinción".

La identidad colectiva se descubre puesta en peligro (injustice frame), lo que desencadena un choque entre lo que es y lo que debería ser el mundo, suscitando sentimientos de indignación e injusticia (hot cognitions) contra 
estas históricas y sistemáticas situaciones de explotación, agravios y discriminaciones que sufren. Esta "estrategia de dramatización" arranca de la combinación de las estructuras cognitivas y simbólicas (Eder, 1992) tradicionales indígenas y de un momento de "liberación cognitiva" (McAdam et al., 1999) que alumbra la construcción de una percepción política en torno a la gravedad de esta situación.

Las causas que han arrastrado a este escenario son consideradas por ellos como el producto de las fuertes agresiones externas que han recibido a lo largo de la historia. Desde el punto de vista de la percepción política indígena, la escasa ética en las prácticas depredadoras, devastadoras e ilegales de estos "agentes", los convierte en los principales responsables y culpables del desequilibrio ambiental que puede conducir al exterminio y desaparición de este pueblo.

El objetivo, por tanto, es garantizar la reproducción y supervivencia del pueblo indígena por medio de la puesta en marcha de políticas públicas que garanticen dos elementos básicos: el reconocimiento y el respeto de su territorialidad, a través de la titulación de sus tierras.

Para ellos, el reconocimiento que han recibido por parte de investigadores de universidades nacionales e internacionales, museos extranjeros, grupos de intelectuales, investigadores, técnicos y profesionales de la cooperación para el desarrollo confirma la importancia de sus costumbres atávicas, tradiciones, conocimientos patrimoniales y creencias ancestrales.

Este marco se dirige especialmente a tres audiencias: la población indígena, en la que prevalece una identidad étnica precaria y frágil; los ciudadanos y autoridades gubernamentales y, por último, las organizaciones de cooperación para el desarrollo, el auditorio más importante. El impacto de este marco en estas tres audiencias conforma una importante parte de la eficacia y el éxito de la movilización indígena.

La estructura del segundo frame (el marco territorialambiental) (gráfica 3, p. 222) se compone de varias dimensiones y estrategias (Rivas, 1993: 209). Por un lado, la protección medioambiental de su hábitat pasa a ser la médula espinal de su discurso, que se resume en dos peticiones concretas a las autoridades gubernamentales: declaración de las reservas de biosfera indígena en sus territorios y la gestión de sus recursos naturales.

¿Cuáles son las causas que generan esta solicitud? Algunos elementos que ponen en peligro la conservación de este rico espacio natural y de sus habitantes: la biopiratería, instalación de centrales hidroeléctricas, invasiones de colonos, extracción de maderas nobles, contaminación

\section{Gráfica 2. Esquema del marco identitario}

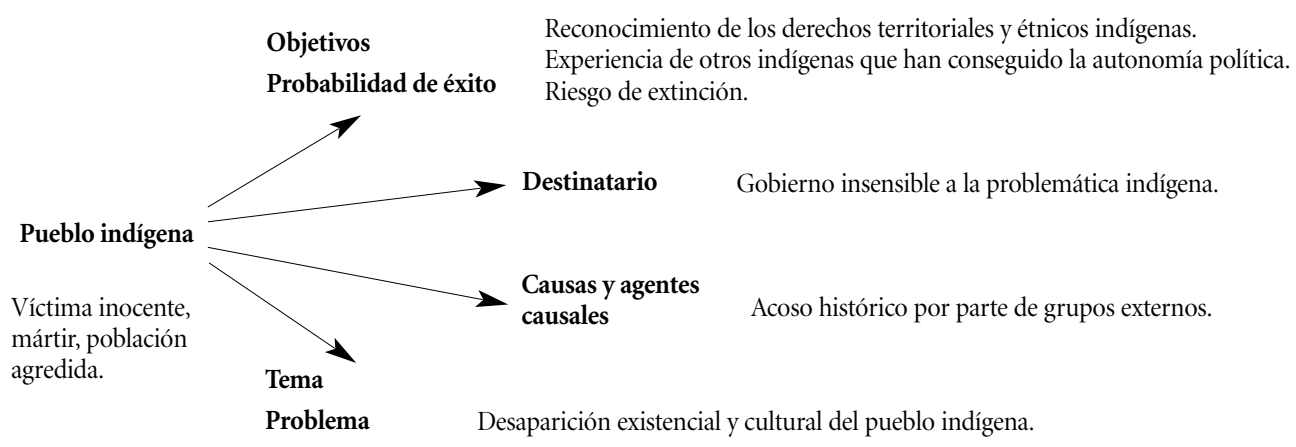

Aliados de prestigio: universidades, ONU, cooperación internacional, etcétera. 
de las aguas, etc. Los agentes culpables de esta amenaza son los campesinos y ganaderos que intentan extender sus actividades en este territorio, las firmas madereras y las diversas compañías que explotan los recursos endógenos.

De todo esto se derivan unos objetivos concretos por parte del movimiento indígena: la búsqueda de garantías legales para el control y gestión sostenible de la zona por parte de sus habitantes. Los éxitos que reseña el discurso indígena se refieren a la experiencia exitosa de otros grupos en la declaración de biosferas en sus territorios. Los destinatarios de la protesta son los gobiernos de cada país. Por último, el discurso autolegitimador de esta reivindicación se basa en el tradicional papel del pueblo indígena como "guardianes y especialistas en el cuidado y mantenimiento de su entorno natural" y como sus mejores conocedores.

El discurso de las organizaciones políticas indígenas intenta hacer compatible las características étnicas de su pueblo con el marco ambientalista que construye. La creencia en la "pacha mama" o "panpas" es compatible con el discurso ecológico radical dominante en los países occidentales, que para autores como Dobson (1997) representa una corriente reciente que cuestiona el "consenso productivista dominante"3. Esta va a ser una de las claves de la fuerte resonancia que esta ideología indígena encuentra en las organizaciones y movimientos ecologistas internacionales.

El tercer frame (marco étnico-cultural) (gráfica 4, p. 222) intenta legitimar las acciones colectivas encaminadas a garantizar la conservación de la unidad del pueblo indígena por medio del desarrollo de la conciencia etnocultural con el fin de fortalecer el impulso unitario sin distinción de sexo, edad, religión ni línea ideológica a la que se pertenezca. La estructura de este tercer frame se vincula con el proceso de reconstrucción de la memoria colectiva, es decir, la conjunción entre la selección y reinterpretación de los acontecimientos históricos y la creación

\footnotetext{
${ }^{3}$ Esta visión global en torno a la tierra y sus elementos se expandió a raíz de la emergencia de la llamada "Hipótesis Gaia", popularizada por James Lovelock en 1986 y que se relaciona con la primera imagen que se toma del total de la tierra en el año 1968 por los ocupantes del Apolo VIII (Dobson, 1997: 37; Dryzek, 1997: 5).
}

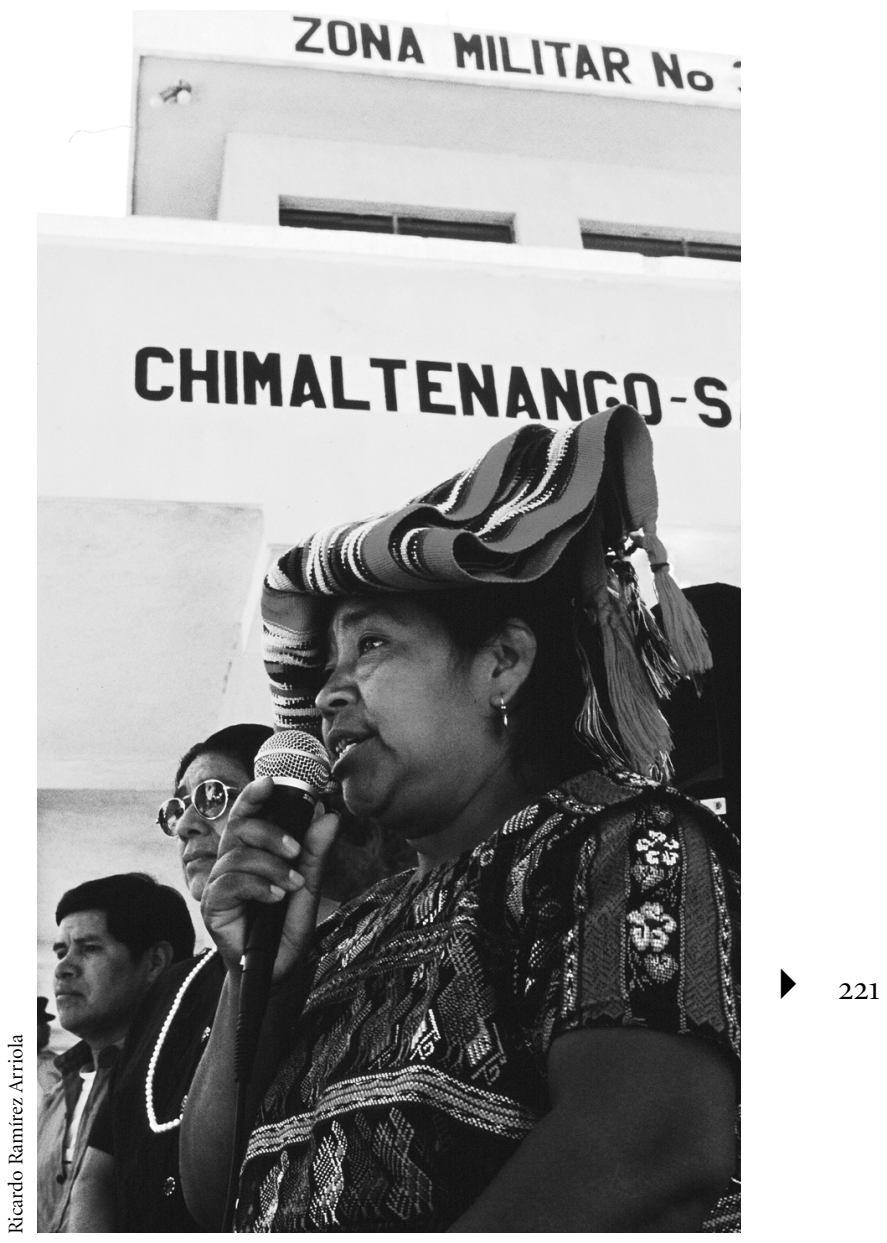

Rosalina Tuyuc, dirigente de Conavigua, durante el acto de devolución de la Zona Militar 302, Chimaltenango, Guatemala, 2002.

imaginaria. Las formas de las redes simbólicas y culturales del discurso étnico-cultural trasmiten y reproducen imágenes, evocando un pasado glorioso y símbolos dramáticos de pueblo errante, víctima de abusos, injusticias y catástrofes de todo tipo, acontecimientos que han provocado sentimientos de rebeldía e indignación entre su población y, sobre todo, entre los líderes, que alientan la formación de la identidad colectiva a modo de unidad frente a la fatalidad del destino.

Por medio de la recuperación de la memoria etnohistórica se intenta consolidar la identidad de la etnia indígena y contribuir a la formación de una auténtica conciencia 
Gráfica 3. Esquema del marco territorial-ambientalista

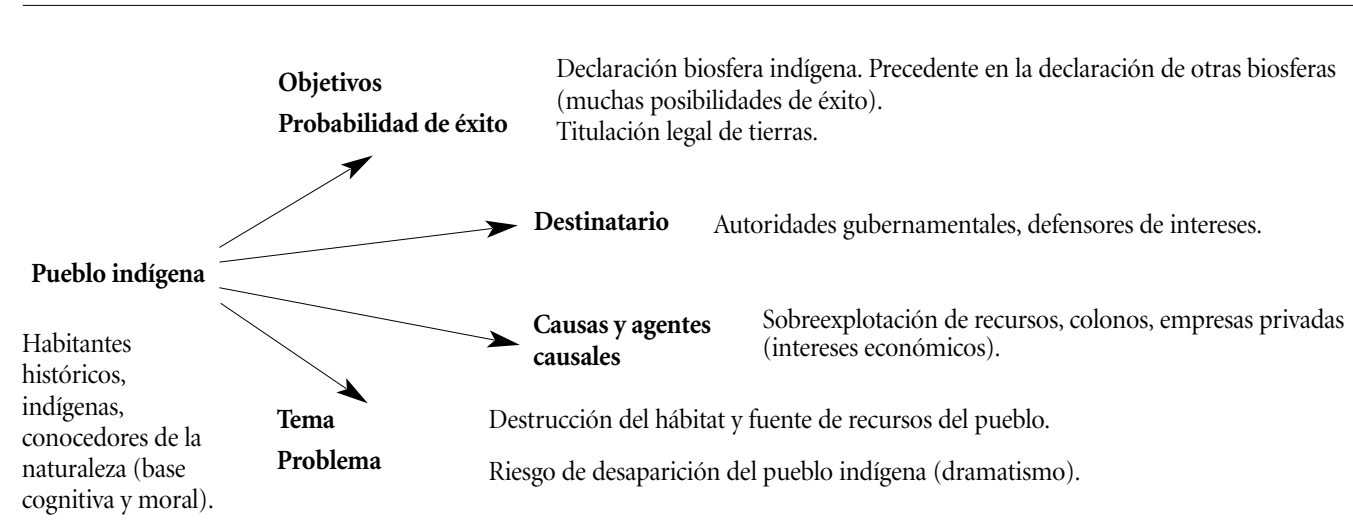

FUENTE: Elaboración propia, con base en el esquema de Gerhards (1995).

Gráfica 4. Esquema del marco étnico-cultural

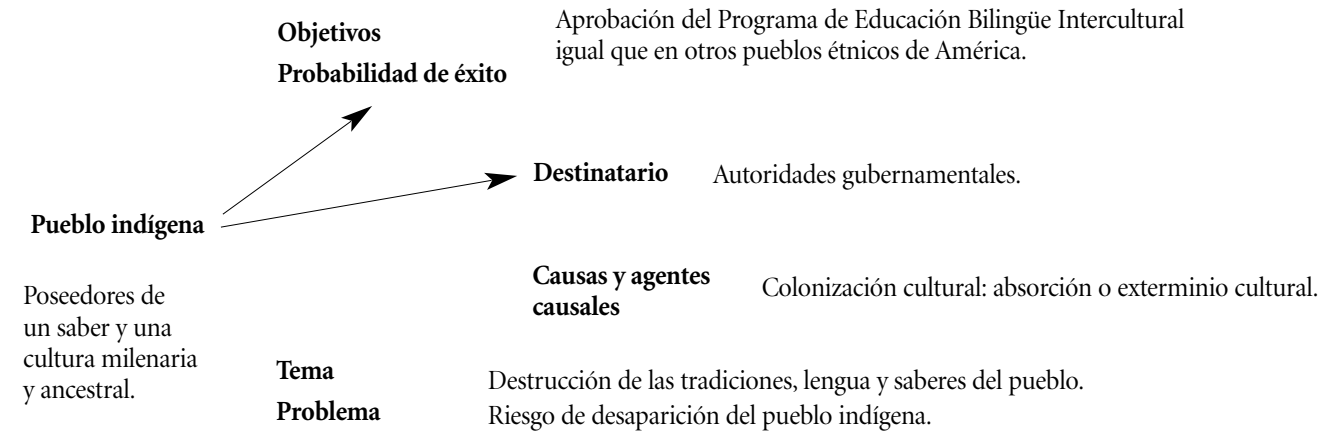

FUENTE: Elaboración propia, con base en el esquema de Gerhards (1995).

social y política, revalorizando y dinamizando la lengua indígena a partir de la difusión y educación bilingüe y multicultural, y mediante la custodia, conservación y administración de los aspectos arqueológicos encontrados en el territorio de este pueblo. La lengua, como expresión directa de una cultura, se convierte en la trinchera de la resistencia cultural, en el refugio del significado identificable (Castells, 1998).

Este discurso político intenta introducirse en las agendas políticas de gobiernos, organismos internacionales y organizaciones de desarrollo para que tomen en cuenta las reivindicaciones planteadas para paliar esta problemática. El Programa de Educación Bilingüe e Intercultural, que supuso uno de los objetivos más importantes del movimiento indígena, se aplicó en algunas comunidades latinoamericanas en sus inicios para la promoción de este tipo de modelo multicultural de educación. Este programa pretende rescatar y recuperar la lengua propia y contribuir a la perdurabilidad de su cultura.

Por otro lado, sus élites políticas, en las declaraciones 
Gráfica 5. Esquema del marco globalicrítico

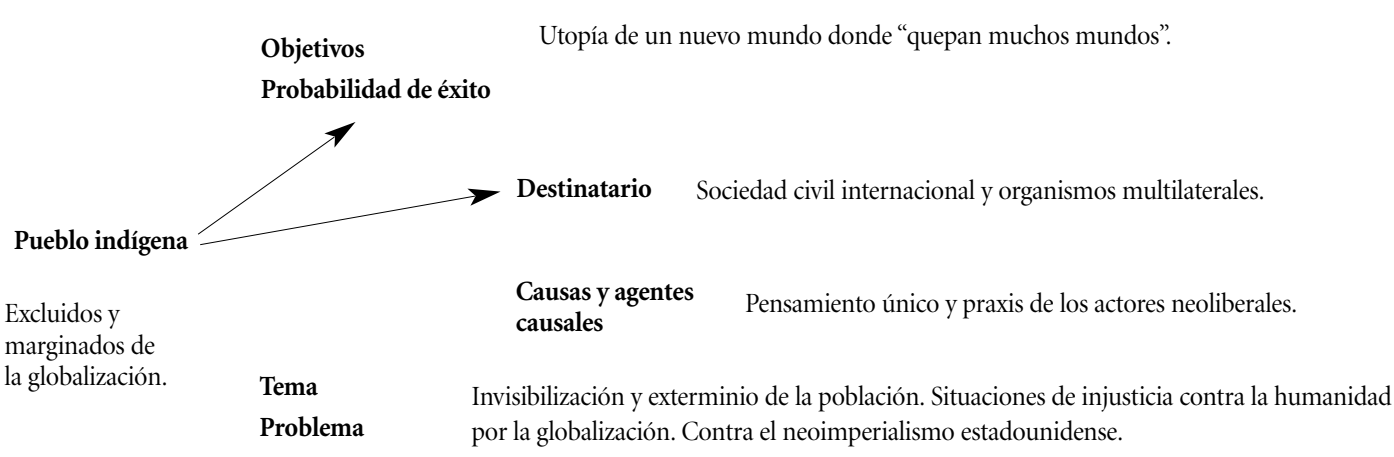

FUENTE: Elaboración propia, con base en el esquema de Gerhards (1995).

públicas y escritos publicados, juzgan a las políticas indigenistas "incorporativas" de la administración gubernamental, y a la dominación cultural histórica que han padecido como factores que también dañan y resquebrajan el ancestral patrimonio cultural y lingüístico indígena.

Para los líderes políticos indígenas, el papel que desempeñan las comunidades y su movimiento social debe ser protagónico en la resolución de estos graves conflictos que ponen en peligro su subsistencia como civilización, pues además de considerarse los más calificados para garantizar el mantenimiento de la riqueza ecológica de este medio ambiente, son los que pueden preservar la infinita riqueza de su saber ancestral y milenario, algo que beneficiaría al patrimonio natural y cultural del conjunto de la humanidad.

En el cuarto marco considerado ("narración globalicrítica") (gráfica 5), las movilizaciones indígenas inciden en el carácter universal de su movilización. Consideran que los movimientos indígenas no pueden reducir su lucha a cuestiones localistas, pues forman parte de la actual estructura económica y sociopolítica global (Sánchez, 1994), la interacción global-local se presenta como inevitable.

En estos discursos, los indígenas identifican a un único actor público: la humanidad, todos los seres humanos excluidos, marginados e invisibilizados por el proceso de globalización o neoliberal. Un gran "nosotros" conformado por las minorías que abarcan "todos los colores de la tierra"4, que engloba a todos aquellos que critican y padecen los efectos devastadores de los nuevos desarrollos socioeconómicos mundiales. Frente a estos enemigos neoliberales, representados por los poderosos como Estados Unidos y sus actuales políticas neocolonizadoras en Afganistán e Irak, el Fondo Monetario Internacional (FMI), el Banco Mundial (BM) o la Organización para la Cooperación y el Desarrollo Económicos (OCDE), y frente al pensamiento único (Ramonet, 1998) vinculado a estas nuevas prácticas de mercado. El movimiento indígena se erige, entonces, como la voz que propone una nueva utopía, un nuevo mundo "donde quepan muchos mundos" construido sin exclusiones y con la participación de toda la realidad plural.

Desde los primeros momentos se comienza a gestar un frente amplio, un movimiento disperso e inclasificable de dimensiones mundiales, que encontrará el posterior relevo en las sucesivas manifestaciones mundiales globa-

\footnotetext{
${ }^{4}$ En un cuento así lo narra el subcomandante Marcos: "y se subieron [los dioses] al copete de La Ceiba y desde ahí empezaron a aventar los colores así nomás y el azul se quedó parte en el agua y parte en el cielo [... y y el café, que era más pesado, se cayó en la tierra, y el amarillo, que era una risa de niño, voló hasta pintar el sol, y el rojo llegó a la boca de los hombres y de los animales y lo comieron y se pintaron de rojo por dentro $[\ldots]$ ni se fijaban dónde llegaba el color que avientan y algunos colores salpicaron a los hombres y por eso hay hombres de distintos colores y distintos pensamientos" (Subcomandante Marcos y Domínguez, 1999).
} 

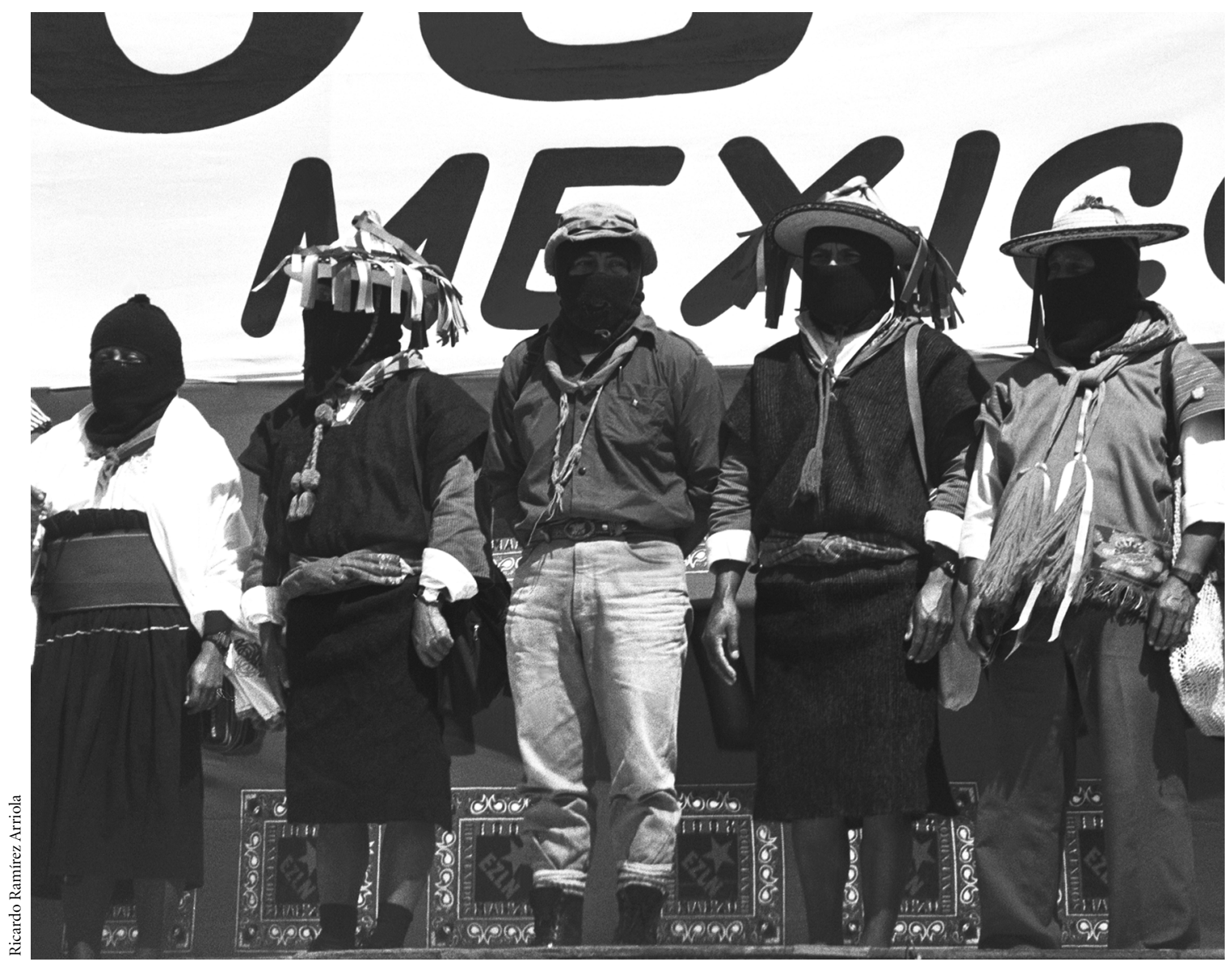

Comandantes zapatistas en el Zócalo de la ciudad de México, marzo de 2001.

lifóbicas y en los foros sociales mundiales. Este movimiento gestado desde la red de redes (Internet) aglutina a una multiplicidad ideológica, cuyo denominador común se concreta en la "solidaridad cósmica", en la "retórica de la diferencia" y en las críticas sistematizadas a los postulados del modelo globalizador imperante. Esta red mundial se ha activado recientemente, a raíz de la oposición contra la invasión a Irak por parte de Estados Unidos, Gran Bretaña y sus aliados, y que aglutinó a millones de personas en manifestaciones callejeras por todo el mundo.

Si analizamos los marcos interpretativos y las organizaciones mentales que llevan a los movimientos indígenas a definir su situación, se observa que han articulado un nuevo discurso político que persigue crear o recrear la autoestima de los grupos de estatus minoritarios mediante la afirmación de su identidad colectiva. Las indicaciones en torno a los problemas sociales y sus causas, los objetivos y la caracterización del destinatario de sus protestas, sirven para justificar a estos movimientos como actores legítimos de las mismas.

La estrategia del movimiento indígena ha sido la definición e interpretación de sus problemas sociales y de las injusticias históricas con el fin de convencer a una gran audiencia de masas y élites de referencia diversas de la necesidad de emprender acciones colectivas y de apoyo a los objetivos de su lucha, por medio de la financiación de políticas públicas y de proyectos de cooperación al desarrollo destinados a solventar sus problemas. 


\section{COMUNICACIÓN DE LOS MARCOS DE INTERPRETACIÓN. EL IMPACTO EN LAS AUDIENCIAS: RESONANCIASY ALINEAMIENTOS}

Para que un proceso de enmarcamiento sea eficaz y consiga el éxito proyectado por el actor social es necesario que se generen alineamientos y resonancias con las audiencias a las que, explícita e implícitamente, va destinado (Snow y Benford, 1993; Gamson, 1990).

En primer lugar, se evalúan los impactos de los procesos de enmarcamiento dentro del propio movimiento indígena. En este caso, los alineamientos del marco deben relacionar las interpretaciones de los individuos (intereses, valores y creencias) con las disquisiciones de la organización que estructura y dirige el movimiento social (ideología, actividades y objetivos). La estrategia de enmarcamiento interna pretendió generar una representación de la identidad política dentro de las propias comunidades, mediante los intentos de localización de alineamientos entre el mundo de vida de cada uno de los indígenas y el mensaje de la organización.

Se produjeron conexiones (frame brigding) (Snow y Benford, 1993) entre los marcos identitario, territorialambientalista y étnico-cultural, generados por la organización política indígena, y las estructuras cognitivas y simbólicas colectivas de este pueblo. La fidelidad narrativa en los relatos históricos y actuales frente al acoso externo; las concepciones animistas y panteístas de la relación hombre-naturaleza, y las intromisiones culturales y religiosas sobre sus creencias y costumbres se asemejan a los mundos de vida que cada día experimentan cotidianamente los propios indígenas, lo que favorece la credibilidad empírica de los marcos generados desde la organización.

En segundo lugar, se calculan los efectos de estos marcos políticos indígenas en la opinión pública nacional de cada país. Definitivamente, los intentos de sensibilización sobre la problemática indígena han sido un modesto triunfo. Por un lado, el escaso eco que las cuestiones étnicas e indígenas mantienen en los medios de comunicación de masas del país, que únicamente cubren las noticias sobre la problemática indígena cuando se acerca el 12 de octubre, cuando hay una exposición de artesanías indí- genas o cuando se produce algún tipo de catástrofe. Por el otro, la configuración de la conciencia nacional construida de espaldas al elemento indígena, negándolo e invisibilizándolo en su inconsciente colectivo histórico, en el imaginario nacional y en los discursos políticos oficiales. Si a esto se le añade el componente ideológico neoliberal dominante en la sociedad latinoamericana, que propugna la abolición de los obstáculos a cualquier iniciativa privada empresarial, se deduce que el choque entre este ícono político (Klandersman et al., 1999) y el marco ambientalista indígena resulta de difícil conciliación.

Las características del sistema político latinoamericano, con una democracia liberal escasamente estabilizada y resultado de una transición autoritaria de pactos entre élites, minimizan el componente participativo de la sociedad civil en la vida pública del país (Tangermann, 1995; Alcántara, 1999). Las orientaciones de la opinión pública no disfrutan del mismo peso político que en los sistemas democráticos más consolidados, por lo que los debates que se generan en ella no originan efectos en las dinámicas políticas del gobierno, lo que desemboca en un agrietamiento entre el sistema político y la sociedad, y multiplica un panorama democrático inestable y precario, con fuertes crisis de gobernabilidad.

Otra audiencia con la que sí han conseguido el éxito deseado la componen los organismos internacionales y las agencias de cooperación para el desarrollo. Los cambios en el sistema cognitivo mundial dan paso a una multiplicidad ideológica cuyo eje común se concentra en la reivindicación del derecho a tener derechos (Stavenhagen, 1996). Estos organismos —Programas de las Naciones Unidas para el Desarrollo (PNUD), Organización de los Estados Americanos (OEA), Banco Interamericano de Desarrollo (BID), BM, etc. - han generado un marco jurídico de protección de los derechos indígenas ${ }^{5}$ y una

\footnotetext{
${ }^{5}$ Las leyes internacionales que amparan los derechos de los pueblos indígenas tienen sus antecedentes en la Declaración Universal de los Derechos Humanos, de 1948. La Convención Internacional sobre la Eliminación de todas las formas de Discriminación Racial fue adoptada por la Asamblea General en 1965. El Fondo de Contribuciones Voluntarias para las Poblaciones Indígenas de las Naciones Unidas fue establecido en 1985, con el objetivo de elaborar el Estudio del Problema de la Discriminación contra las Poblaciones Indígenas. En 1989,
} 
"institucionalización" de las únicas políticas sociales que se desarrollan en países con gobiernos tan endeudados y dependientes, sin capacidad de autogestión, marcando así las políticas de integración social con sus propios programas, o bien, financiando las partidas presupuestarias de los ministerios, que actúan asesorados por los lineamientos que sus entidades financieras les marcan.

El marco de interpretación ambientalista indígena encuentra fuertes resonancias en el marco ecologista (Dobson, 1997; Dryzek, 1997; Eder, 1992), en el que se sitúan muchas organizaciones de cooperación al desarrollo, organismos internacionales, asociaciones ecologistas, intelectuales y profesionales del medio ambiente. Este master frame se genera en la década de 1970, fruto de la creciente asunción de los límites ecológicos, y creó un clima pesimista que alimentó una "nueva nostalgia de una forma de vida simple y natural, y desencadenó una oleada de críticas generalizadas a la burocracia y la industrialización" (Gamson y Meyer, 1999: 396). Se centró en la calidad de vida y llegó a componer un nuevo marco para todos los órdenes de la vida política y social (Eder, 1992).

Las perspectivas ecologistas van a utilizar como modelos ideales de referencia a las formas de vida típicas de las comunidades indígenas ${ }^{6}$.

la Organización Internacional del Trabajo (OIT) revisó el artículo 107 sobre "Poblaciones indígenas y tribales" del año 1957, y en 1989 introdujo el Convenio 169: "Convenio sobre los Pueblos Indígenas y Tribales". La celebración del "Año Internacional de los Pueblos Indígenas" (1993) por las Naciones Unidas, y la Década Internacional de los Pueblos Indígenas reestablecida por la ONU (1995-2004) son una muestra más del interés por proteger a estos pueblos. La Comisión Interamericana de Derechos Humanos fue creada por la OEA, en 1954, para la protección de los derechos humanos en el marco de la Convención Americana. La Cumbre Iberoamericana de Jefes de Estado de 1992 creó el Fondo para el Desarrollo de los Pueblos Indígenas de América Latina y el Caribe. El Banco Mundial emprendió, en 1991, el diseño de la Directriz Operativa sobre Pueblos Indígenas, en la que se potencia la participación y beneficio de los indígenas en sus proyectos. El BID institucionaliza, en 1990, un control de calidad en cuanto a los posibles impactos sobre poblaciones indígenas. En 1995, la Unión Europea aprobó una estrategia de ayuda pública al desarrollo destinada a los pueblos indígenas. Anteriormente, el Consejo y el Parlamento habían realizado varias resoluciones acerca de los pueblos indígenas (Gómez, 2001; Van de Fliert, 1997)

${ }^{6}$ Esto se refleja en los análisis del denominado "paquete fundamentalista", descrito por Klaus Eder, para caracterizar los movimientos ecologistas en Europa (1992: 18); también en los trabajos sobre la ideología ecologista radical, analizados por Dobson $(1997: 28,115)$ y, por
Estos nuevos actores sociales internacionales van a estar insertos en los polos ideológicos posadquisitivos, culturalistas y posmaterialistas ${ }^{7}$, que intervienen como constructores de las solidaridades internacionales, y que van a compartir un master frame gestado a partir del movimiento mundial contra la celebración del quinto centenario del descubrimiento de América, que reunió los íconos políticos anticoloniales con los nuevos discursos democratizadores, de respeto a los derechos humanos y de apoyo a las minorías excluidas y marginadas, y que apoyaran la causa indígena por sus componentes de identidad, etnicidad, ecologismo y defensa de los derechos de una minoría desfavorecida.

Los indígenas, al igual que el resto de los movimientos étnicos, han combatido por conseguir el ingreso en el espacio político y por su reconocimiento como sujetos sociales modernos, representantes de nuevos intereses colectivos, reivindicando con orgullo la figura del indígena, que nunca estuvo incluida en el imaginario patrio y en la identidad nacional.

\section{CONCLUSIONES}

De esta reflexión se desprende que las acciones colectivas indígenas se construyen y desarrollan no sólo cuando las condiciones materiales de existencia son extremas y su marginación y discriminación política es acentuada, sino cuando estos grupos humanos encuentran un contexto político favorable, cuando las tácticas y estrategias seleccionadas para publicar, extender y conseguir sus reivindicaciones son eficaces y cuando el discurso político e ideológico que manejan para legitimarse y justificar sus

supuesto, en los estudios en torno al "radicalismo verde" en su versión romántica, definida por Dryzek (1997: 155-159).

${ }^{7}$ Este concepto, acuñado por Ronald Inglehart, ha generado fuertes polémicas en el ámbito de las ciencias sociales. Se considera que el modelo que propone este autor resulta impreciso en varios aspectos: los resultados de la investigación de campo en torno a los valores dominantes en la población europea no son fiables y generalizables y la unidireccional del paso de valores materialistas a posmaterialistas en sociedades tardomodernas no es adecuada pues se ha constatado la existencia de estos valores en sociedades y grupos sociales pobres (Inglehart, 1991; Riechmann y Fernández Buey, 1999: 31). 


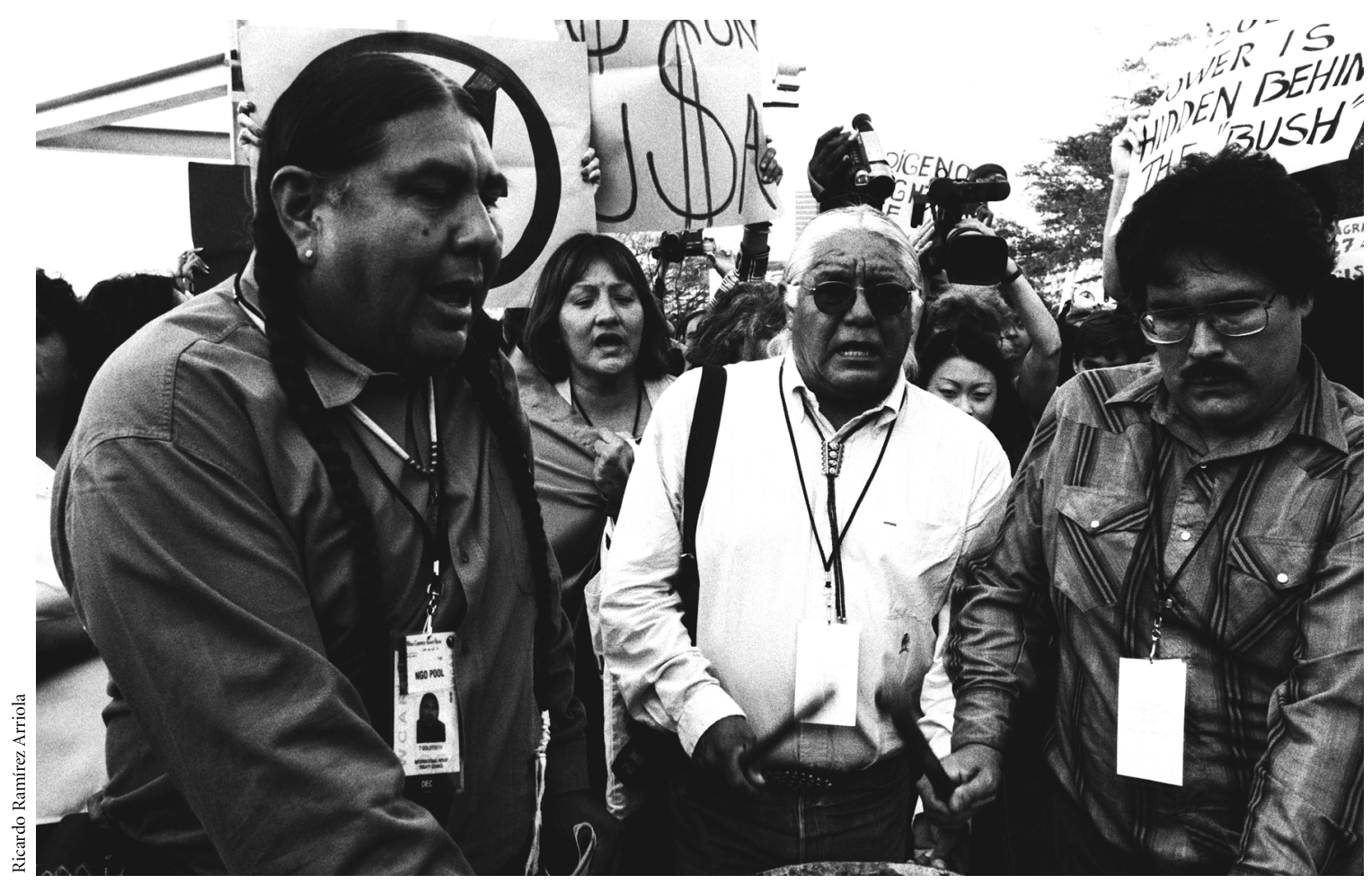

Cónclave de los delegados de los pueblos indígenas en la Conferencia Mundial contra el Racismo, la Discriminación Racial, la Xenofobia y las Formas Conexas de Intolerancia, Durban, Sudáfrica, 31 de agosto-8 de septiembre de 2001.

derechos políticos recibe fuerte aceptación y apoyo entre ciertas élites dominantes en ese momento.

La extrapolación de las conclusiones desprendidas de la experiencia indígena al resto del universo de los movimientos étnicos mundiales estrecha la selección de las variables que deben tenerse en cuenta al analizar las causas de la construcción, desarrollo y consolidación de una movilización política de carácter étnico. Los factores como el contexto de oportunidad política que pueden disfrutar, optimizar y regenerar; las estrategias y tácticas utilizadas en acciones colectivas y movilizaciones; y unos discursos ideológicos resonantes entre ciertos actores sociopolíticos claves e influyentes, las variable que se han desarrollado en este artículo son fundamentales para apoyar y favorecer la constitución de cualquier movilización indígena y para alcanzar los objetivos políticos perseguidos.

La ideología del movimiento indígena, desarrollada en sus discursos y prácticas, se ubica principalmente dentro del llamado paradigma del reconocimiento o multiculturalismo, centrado en el campo de los valores culturales, sociales y políticos. Manejan un lenguaje político forjado en torno a la categoría de la identidad y dotado de su propio repertorio retórico y terminológico. En términos generales, aluden a una postura moral y políticamente favorable al pluralismo cultural y a los modelos de integración social y de gestión política que persigan su fomento. Lo que se reivindica es el derecho a la disparidad y el orgullo de la identidad diferencial.

En Latinoamérica, los movimientos étnicos fraguados desde la década de 1980 han representado una nueva forma del quehacer político y han desplegado inéditos conductos de participación social. Las alteraciones de los sistemas políticos que los nuevos movimientos étnicos consigan formalizar pueden generar un procedimiento sociopolítico que origine el nacimiento de un nuevo melting pot social y de un estado multicultural y pluriétnico. 
Las preguntas inconclusas de esta reflexión son múltiples y tan sólo se consiguió la aproximación a alguna de ellas. Las nuevos interrogantes que se pudieran abordar quedan abiertos para las próximas investigaciones de los científicos sociales: ¿̇los movimientos étnicos modificarán el panorama de los sistemas democráticos en América Latina y del mundo?; ¿asistiremos a un cambio radical en el panorama ideológico mundial?; ¿qué nuevas fórmulas adoptarán los próximos movimientos étnicos?, y ¿qué funciones van a ejercer los organismos de cooperación para el desarrollo y al "tercer sector" en los países con fuerte dependencia externa? Estas incógnitas moldean las profundas incertidumbres que depara el incierto futuro, en esta época de tardomodernidad que inaugura este nuevo milenio.

\section{Bibliografía}

Alcántara, M., 1999, Sistemas políticos de América Latina. México, América Central y el Caribe, vol. II, Tecnos, Madrid.

Anderson, B., 1993, Las comunidades imaginadas, Fondo de Cultura Económica, México.

Bourdieu, P., 1988, La distinción, Madrid, Taurus.

Castells, M., 1998, La era de la información. Economía, sociedad y cultura. Vol. 2. El poder de la identidad, Alianza, Madrid.

Cohen, J. L. y A. Arato, 2000, Sociedad civil y teoría política, Fondo de Cultura Económica, México.

Delgado y Gutiérrez (eds.), 1999, Métodos y técnicas cualitativas de investigación en ciencias sociales, $3^{\text {a }}$ ed., Síntesis, Madrid.

Dobson, A., 1997, Pensamiento politico verde, Paidós, Madrid.

Dryzek, J., 1997, The Politics of the Earth: Environmental Discourses, Oxford University Press, Oxford, Reino Unido.

Eder, K., 1992, Framing and Communicating Environmental Issues. A Discourse Analysis of Environmentalism, Instituto Universitario Europeo, Florencia.

Gamson, W., 1990, The Strategy of Social Protest, Wadsworth Publications, California.

— y D. S. Meyer, 1999, "Marcos interpretativos de la oportunidad política", en D. McAdams, J. McCarthy y M. Zaid, Movimientos sociales: perspectivas comparadas, Istmo, Madrid.

Gerhards, J., 1995, Framing Dimensions and Framing Strategies: Contrasting Ideal-and Real-type Frames, Social Science Information, Londres.
Goffman, E., 1974, Frame Analysis, Harvard University Press, Cambrigde.

Gómez, A., 2001, Indigenismo y movilización politica en América Latina: los tawahkas, Universidad de Santiago de Compostela, Santiago de Compostela.

— 2004, Patrones de movilización política de la acción indígena zapatista: contextos, estrategias y discursos, Universidad de Vigo, Vigo.

—, M. Vázquez y S. Leetoy, 2004, Guerrilla y comunicación. La propaganda politica del EZLN (1994-2004), La Catarata, Madrid.

Gurr, T. R., 1995, Minorities at Risk: A Global View of Ethnopolitical Conflicts, Institute of Peace Press, Washington.

Ibarra, P. y B. Tejerina (comps.), 1998, Los movimientos sociales, Trotta, Madrid.

Inglehart, R., 1991, El cambio cultural en las sociedades industriales avanzadas, Paidós, Barcelona.

Klandermans et al., From Structure to Action: Comparing Social Movements Research Across Cultures, Jai Press, Connecticut y Londres.

León, E. y H. Zemelman, 1998, Subjetividad: umbrales del pensamiento, Antrophos, Barcelona.

Lévi-Strauss, C., 1964, Mitológicas III. El origen de las maneras de la mesa, Siglo XXI, Madrid.

Máiz, R., 1995, "La construcción de las identidades políticas", Revista Vasca de Sociología y Ciencia Política, "Inguruak", núm. 13, pp. 9-23.

McAdams, D., J. McCarthy y M. Zald (comps.), 1999, Movimientos sociales: perspectiva comparada, Istmo, Madrid.

Rivas, R., 1993, Pueblos indígenas y garífuna de Honduras, Guaymuras, Tegucigalpa.

Ramonet, I., 1998, Geopolitica do caos, Vozes, Petrópolis.

Riechmann, J. y F. Fernández Buey, 1999, Redes que dan libertad, Paidós, Barcelona.

Sánchez, C., 1994, La conformación étnico-nacional en Nicaragua, Instituto Nacional de Antropología e Historia, México (Col. Científica).

Snow, Hunt y Benford, 1993, Framing Processes and Identity Construction in Collective Action, presentado en la reunión anual de la Midwest Sociological Society, Chicago.

Stavenhagen, R., 1996, Ethnic Conflicts and the NationState, UNRISD, Nueva York

Subcomandante Marcos y Domitila Domínguez, 1999, La historia de los colores, Colectivo Callejero.

Tangermann, C. (comp.), 1995, Ilusiones y dilemas. La democracia en Centroamérica, FLACSO, Costa Rica.

Tarrow, S., 1997, Power in Movement. Social Movements, Collective Actions and Cycles of Protest, Cornell University Press, Cornell.

Van de Fliert, L., 1997, Guía para los pueblos indígenas, Comisión Nacional de Derechos Humanos, México. 